\title{
Outcome of Laparoscopic Cholecystectomy as Ambulatory Surgery: Experience of 250 Cases
}

\author{
Ullah ME ${ }^{\mathrm{a}}$, Rahman $\mathrm{MM}^{\mathrm{b}}$, Rabbi ${ }^{\mathrm{c}}$, Alam MNA ${ }^{\mathrm{d}}$, Maitra TK
}

\begin{abstract}
Background: Laparoscopic cholecystectomy has been performed as outpatient procedure for many years. Few studies have been conducted with primary focus on patient acceptance and preferences in terms of safety and satisfaction. We tried to explore its feasibility in otherwise healthy individuals undergoing laparoscopic cholecystectomy.

Methods: Data from 250 patients with cholelithiasis who underwent laparoscopic cholecystectomy in BIRDEM General Hospital from April 2017 to June 2018 as ambulatory surgery (hospital stay d"23 Hours) with or without overnight stay were analyzed. Complications, admissions and readmissions, patient satisfaction and treatment expenditure were assessed.

Results: Mean duration of hospital stay was 17.3 hours. Two patients had complications (deep port site infection) on $5^{\text {th }}$ post-operative day after surgery. Only $2.4 \%$ patients required readmission while $9.2 \%$ patients had unplanned admission. Seventy six point four percent patients were highly satisfied with the procedure. Treatment cost was about $15 \%$ lower than routine inpatient operation.
\end{abstract}

Conclusion: Laparoscopic cholecystectomy can be performed as an outpatient procedure with a low rate of complications and admissions/readmissions. Patient acceptance in terms of satisfaction is high.

(BIRDEM Med J 2019; 9(2): 157-161)

\section{Introduction}

Anesthetic and medical advances and particularly recent surgical technological developments have encouraged healthcare providers to explore the option of carrying out a signiûcant proportion of surgical procedures on

\section{Author Information}

a. Dr. Mahmud Ekram Ullah, Assistant Professor, Dept. of Surgery, BIRDEM General Hospital \& Ibrahim Medical College, Dhaka

b. Dr. Md. Mushfiqur Rahman, Associate Professor, Dept. of Anesthesia, BIRDEM General Hospital \& Ibrahim Medical College, Dhaka

c. Dr. Hashim Rabbi, Associate Professor, Dept. of HBP surgery, BIRDEM General Hospital \& Ibrahim Medical College, Dhaka

d. Prof. Md. Noor A Alam, Professor, Dept. of Surgery, BIRDEM General Hospital \& Ibrahim Medical College, Dhaka

e. Prof. Tapash Kumar Maitra, Professor and Head, Dept. of Surgery, BIRDEM General Hospital \& Ibrahim Medical College, Dhaka

Address of correspondence: Dr. Mahmud Ekram Ullah, Assistant Professor, Dept. of Surgery, BIRDEM General Hospital, 122 Kazi Nazrul Islam Avenue, Shahbag, Dhaka 1000. E-mail: drmahmud50@gmail.com

Received: February 14, 2019

Accepted: February 28, 2019 an outpatient basis. The continued improvement in anesthesia techniques, such as regional anesthesia, ultrashort acting drugs with minimal side effects, more relevant ambulatory discharge criteria and minimal invasive surgery techniques will allow larger numbers of patients to take advantage of ambulatory surgery. ${ }^{1,2}$ A number of studies have documented the safety and feasibility of outpatient laparoscopic cholecystectomy in an ambulatory surgery unit in selected patients. ${ }^{3-6}$ The majority of these studies have focused on outcomes such as complications, admissions and readmissions. There are other aspects to assess: its efficacy like quality of life and patient satisfaction level.

Our hospital has the experience of performing different general surgical, laparoscopic ${ }^{7}$, orthopedic and urological procedures regularly as outpatient basis in good number of cases. Also the high turnover of laparoscopic cholecystectomy in our center inspired us to evaluate its safety and acceptance as outpatient procedure in our set up. Hence we tried to explore its feasibility in otherwise healthy individuals undergoing laparoscopic cholecystectomy. 


\section{Methods}

This observational study was carried out in the Department of Surgery, BIRDEM General Hospital, Dhaka, Bangladesh from April 2017 to June 2018 based on convenient sampling. All patients between the ages of 18 and 50 years, who presented for laparoscopic cholecystectomy were considered for entry into the study based on inclusion and exclusion criteria. Patients with an American Society of Anesthesiologists (ASA) score of III or IV, extreme obesity, patients who underwent upper abdominal surgery before, coagulopathy and patients with acute cholecystitis or small, contracted gall bladder were considered unsuitable for outpatient surgery and were excluded from enrolment. Patients (if any) who needed conversion to open cholecystectomy were also excluded from the study. Furthermore, included patients were required to live less than $10 \mathrm{~km}$ from the hospital. Informed consent was obtained from all participants.

Patients, either reported at the reception of operating room at 8:00 am and operated on early in the morning or alternatively at 5:00 pm and operated in the evening according to operating room schedule. The laparoscopic cholecystectomy procedure was performed by consultant surgeon using the standard four port technique. The perioperative and anesthetic regimens were standardized. Induction was done by propofol 2 $\mathrm{mg} / \mathrm{kg}$, muscle relaxation by atracurium $0.5 \mathrm{mg} / \mathrm{kg}$ and analgesia by fentanyl $2 \mu \mathrm{g} / \mathrm{kg}$. Maintenance was done by propofol 200-300 mg/hour and atracurium $10 \mathrm{mg}$ every 25 minutes interval. Prophylaxis against postoperative pain and nausea was achieved by locally infiltrating combined equal volume of xylocaine $2 \%$ and bupivacaine $0.5 \%$ in all port sites and postoperative administration of $30 \mathrm{mg}$ ketorolac and / or $1 \mathrm{~g}$ paracetamol and $4 \mathrm{mg}$ ondansetron.

Patients were encouraged to mobilize and start oral intake if fully conscious and not nauseated. Discharge was allowed if the patient required oral pain medication only, tolerated oral fluids, had passed urine spontaneously and felt conûdent of managing at home. On discharge, each patient was provided with a 2-day supply of ketorolac/ tramadol (if non-steroidal antiinflammatory drug was contraindicated) and 2 week supply of omeprazole to be taken as prescribed if required.

Recovery from anesthesia was assessed by Aldrete score where consciousness level, respiration, blood pressure, oxygen saturation by pulse oximetry and activity level were measured. Out of 10 , a score of 9 or more indicates complete recovery and is a requirement for discharge.

Efficacy of pain management was assessed by verbal rating score. Patients were instructed to score their pain level from $1-10$, where 10 being the worst pain imaginable and 1 for no pain. Accordingly score between 1-3 means excellent analgesia, 4-6 means fair analgesia and 7-10 means poor analgesia.

Outcome measures included hospital stay, complications if any, admission and readmission rate, operation time, post-operative pain management, nausea, vomiting, wound-related complications and level of patient satisfaction. All patients were contacted over telephone 24 hours following discharge and were asked about their comfort regarding usual daily activity and limitation if any. They were again followed-up at $7^{\text {th }}$ post-operative day at outpatient clinic.

Direct hospital expenditure was calculated from receipts of operation charge (as per hospital procedure charge list), medicine bill, seat rent, laboratory charge (as per hospital investigation charge list) and other utility bill and was compared with that of regular inpatient laparoscopic cholecystectomy.

\section{Results}

A total of 250 patients were included in this study. All patients underwent laparoscopic cholecystectomy under general anesthesia. Eighty-six patients were male; one hundred sixty-four patients were female. Baseline characteristics are shown in Table I.

Table I Base-line characteristics of the study participants $(\mathrm{N}=250)$

\begin{tabular}{ll} 
Parameter & Frequency \\
\hline Age & $15-50$ years, Mean : 30.3 years \\
Sex - more female patients & M: F ratio $-1: 1.91(66.0 \%)$ \\
BMI $\left(\mathrm{kg} / \mathrm{m}^{2}\right)$ & Mean -22.9 (range 19.2 to 23.9$)$ \\
Associated medical conditions & \\
Hypertension & 47 patients $(18.8 \%)$ \\
Diabetes mellitus & 103 patients $(41.2 \%)$ \\
\hline
\end{tabular}


All patients had good anesthetic fitness. Among them $64 \%$ belonged to ASA I and 36\% belonged to ASA II. Regarding airway management, laryngeal mask airway technique (LMA) was used in $30 \%$ cases and endotracheal airway in $70 \%$ cases. Average operating time was 39 minutes (range 26- $73 \mathrm{~min}$ ). Cholecystectomy was completed laparoscopically in 100\% cases, none needed conversion (Table II).

Table II Anesthesia and operation details ( $\mathrm{N}=250)$

\begin{tabular}{ll} 
Parameter & Frequency \\
\hline General anesthesia & $250(100 \%)$ patients \\
ASA I & $160(64.0 \%)$ \\
ASA II & $70(36.0 \%)$ \\
Laryngeal mask airway & $75(30 \%)$ \\
Endotracheal tube & $175(70 \%)$ \\
Conversion to open & $0(0 \%)$ \\
cholecystectomy & \\
Operating time & $26-73$ (mean 39) min \\
\hline
\end{tabular}

Patients recovered very well in the postoperative period. Only six patients had to be readmitted within 7 days of surgery. Two of them were readmitted for deep umbilical port infection. Other patients were readmitted for different medical conditions but there was no mortality in our series. We did not encounter any other major complication. Unplanned admission rate was a bit higher but mainly due to patient preference (concern about transport / traffic) and the remaining due to continued injectable drugs or fluids. Average hospital stay was 17.3 hours.

\begin{tabular}{|c|c|}
\hline Parameter & Frequency \\
\hline \multicolumn{2}{|l|}{$\overline{\text { Recovery }}$} \\
\hline Smooth & $247(98.8 \%)$ \\
\hline Delayed & $3(1.2 \%)$ \\
\hline Major morbidity & $2(0.8 \%)$ \\
\hline Mortality & $0(0 \%)$ \\
\hline Unplanned admission & $23(9.2 \%)$ \\
\hline Readmission (within 7 Days) & $6(2.4 \%)$ \\
\hline Hospital stay & $\begin{array}{l}11-23 \text { hours } \\
\text { (Mean } 17.3 \text { hours) }\end{array}$ \\
\hline \multicolumn{2}{|l|}{ Hospital expenditure } \\
\hline Outpatient & 34,000 BDT (400 USD) \\
\hline Regular inpatient & 40,000 BDT (475 USD) \\
\hline
\end{tabular}

Patient satisfaction level was determined based upon parameters like management of pain and post-operative nausea and vomiting (PONV), quality of life and their comments on reception and discharge facilities. Majority $(73.6-78.8 \%)$ of the patients had excellent experience regarding pain and PONV management, standard of life and accordingly the level of overall satisfaction (76.4\%). However, some patients were not fully satisfied with the reception and discharge facility $(39.6 \%$ and $32.4 \%$ respectively).

Direct hospital expenditure was about 34,000 BDT (400 USD) which is $6,000 \mathrm{BDT}(15 \%)$ lower than inpatient laparoscopic cholecystectomy (40,000 BDT) (475 USD) due to exemption of seat rent and reduction of some service charges. This was apart from indirect expenses that an inpatient incurs from late resumption of economic activity together with that of attending person.

\section{Table IV Patient satisfaction}

\begin{tabular}{lc} 
Parameter & Frequency \\
\hline Pain management & $184(73.6 \%)$ \\
Excellent & $57(22.8 \%)$ \\
Fair & $9(3.6 \%)$ \\
Poor & \\
PONV & $197(78.8 \%)$ \\
Nil or minimal & $48(19.2 \%)$ \\
Mild to moderate & $5(2.0 \%)$ \\
Worse & \\
Reception facility & $151(60.4 \%)$ \\
Excellent & $65(26.0 \%)$ \\
Fair & $34(13.6 \%)$ \\
Poor & \\
Discharge facility & $169(67.6 \%)$ \\
Excellent & $63(25.2 \%)$ \\
Fair & $18(07.2 \%)$ \\
Poor & \\
Quality of life & $186(74.4 \%)$ \\
Excellent & $54(21.6 \%)$ \\
Fair & $10(4.0 \%)$ \\
Poor & \\
Overall satisfaction & $191(76.4 \%)$ \\
Highly satisfied & $52(20.8 \%)$ \\
Well satisfied & $7(2.8 \%)$ \\
Not satisfied &
\end{tabular}




\section{Discussion}

The present study demonstrated that laparoscopic cholecystectomy can be performed as outpatient/ day case procedure without compromising the safety of the patient, as shown in some previous studies. ${ }^{4-6,8-10} \mathrm{In}$ outpatient surgery, patient selection criteria have an impact on the admission/readmission rate. Inclusion criteria were comparatively strict in the present study and perhaps an even larger proportion of patients could be considered for outpatient laparoscopic cholecystectomy. The very small number of readmission indicates that this selection was appropriate and that well informed patients can cope up with some degree of pain and nausea at home.

When a procedure shifts from inpatient to outpatient practice, at first highly selected patients are chosen for the day-case procedure. Eventually patients with some degree of co-morbidity are included and some centers have reported routine laparoscopic cholecystectomy without specific patient selection, at the cost of a higher admission/ readmission rate. ${ }^{11-14}$ Outpatient laparoscopic cholecystectomy has been demonstrated to be safe even for older and high-risk (ASA grade III) patients undergoing elective operations. ${ }^{13}$ However, as we are in the preliminary stage of performing laparoscopic cholecystectomy as outpatient surgery, our policy was to exclude high-risk patients and those with risk factors for anticipated difficult surgery and thus a higher risk of conversion.

As postoperative pain, nausea and vomiting have been reported as significant problems in ambulatory surgical patients, several authors have developed strategies to combat these problems during laparoscopic cholecystectomy. ${ }^{15}$ In the present study an improved anesthetic regimen was used that did not include nitrous oxide and ondansetron was added as antiemetic prophylaxis. Postoperative pain was successfully controlled by multimodal analgesia. These measures seemed to be important in achieving a low postoperative admission/ readmission rate. ${ }^{14}$

In three previous randomized trials of day-case/ outpatient laparoscopic cholecystectomy, the admission rate varied appreciably. In one ${ }^{8}$ it was similar to that in the present study ( $8 \%$ ), whereas admission rates of $26 \%$ and $18 \%$ respectively were reported in the other two trials. ${ }^{9}, 10$ The most likely reason for these higher rates was that strategies to combat postoperative nausea and pain were suboptimal. An important aspect of day case surgery is patient acceptance. In some previous studies of day case laparoscopic cholecystectomy, patient satisfaction varied from 60 to $95 \%{ }^{5,6,8}$ By and large the present results are in line with others, demonstrating similar quality of life outcomes for the management strategies. ${ }^{8,9}$

Over the past decade there has been a push towards performing an increasing number of surgical procedures in the outpatient setting, mainly for economic reasons. ${ }^{16}$ The use of innovative surgical and anesthetic techniques will allow larger numbers of patients to take advantage of elective laparoscopic cholecystectomy on an ambulatory basis. ${ }^{17}$ This has resulted in a rapid shift from inpatient to outpatient practice once good clinical outcome (a safe procedure with no increased risk) and cost effectiveness have been established. Cost reduction in our cases were due to exemption of seat rent and service charges and lower other miscellaneous expenses. However, we faced difficulty to convince some patients for this service due to transport issue and uncertainty of traffic. These issues have to be properly addressed and necessary steps should be taken to carry out majority of our laparoscopic cholecystectomy procedures on outpatient basis.

\section{Conclusion}

It seems reasonable to conclude that laparoscopic cholecystectomy as outpatient surgery provides quality care that is safe as well. Most patients were satisfied with the outcome of surgery because of shorter hospital stay and lower cost. However, there are areas to make further improvement that will increase patient acceptance in terms of satisfaction. Good communication between patient and health care provider will enhance the effectiveness of this service. Gradually its scope can be further extended in the future to include more complicated cases.

Conflict of interest: Nothing to declare.

\section{References}

1. American Society of Anesthesiologist Task Force on Post anesthetic Care. Practice guidelines for Post anesthetic care. Anesthesiology 2002; 96: 742-52.

2. McGrath B, Chung F. Postoperative recovery and discharge. Anesth Clin North Am 2003; 21:367-86.

3. Singleton RJ, Rudkin GE, Osborne GA, Watkins DS, Williams JA. Laparoscopic cholecystectomy as a day surgery procedure. Anaesth Intensive Care 1996; 24: 231-36. 
4. Prasad A, Foley RJ. Day case laparoscopic cholecystectomy: a safe and cost effective procedure. Eur J Surg 1996; 162: 43-46.

5. Mjaland O, Raeder J, Aasboe V, Trondsen E, Buanes T. Outpatient laparoscopic cholecystectomy. Br J Surg 1997; 84: 958-61.

6. Richardson WS, Fuhrman GS, Burch E, Bolton JS, Bowen JC. Outpatient laparoscopic Cholecystectomy : Outcomes of 847 planned procedures. Surg Endosc 2001; 15: 193-95.

7. Ratan MEH, Alam H, Karim MA. Can all benign gallbladder diseases be managed laparoscopically? BIRDEM Med J 2018; 8(1): 35-41

8. Keulemans Y, Eshuis J, de Haes H, de Wit LT, Gouma DJ. Laparoscopic cholecystectomy: day-care versus clinical observation. Ann Surg 1998; 228: 734-40.

9. Dirksen CD, Schmitz RF, Hans KM, Nieman FH, Hoogen boom LJ, Go PM. Ambulatory laparoscopic cholecystectomy is as effective as hospitalization and from a social perspective less expensive: a randomized study. Ned Tijdschr Geneeskd 2001; 145: 2434-39.
10. Hollington P, Toogood GJ, Padbury RT. A prospective randomized trial of day-stay only versus overnight-stay laparoscopic cholecystectomy. Aust N Z J Surg 1999; 69: 841843

11. Taylor E, Gaw F, Kennedy C. Outpatient laparoscopic cholecystectomy feasibility. J Laparo Endosc Surg 1996; 6: 73-77.

12. Fiorillo MA, Davidson PG, Fiorillo M, D'Anna JA Jr, Sithian N, Silich RJ. 149 ambulatory laparoscopic cholecystectomies. Surg Endosc 1996; 10: 52-56.

13. Voitk AJ. Is outpatient cholecystectomy safe for the higher risk elective patient? Surg Endosc 1997; 11: 1147-49.

14. Simpson JP, Savarise MT, Moore J. Outpatient laparoscopic cholecystectomy: what predicts the need for admission? Am Surg 1999; 65: 525-28

15. Kehlet H. Acute pain control and accelerated postoperative surgical recovery. Surg Clin North Am 1999; 79: 431-43.

16. M. Johansson, A. Thune, L.Nelvin, L. Lundell. Randomized clinical trial of day-care versus overnight-stay laparoscopic cholecystectomy. British Journal of Surgery 2006; 93: 40-45

17. Shnaider I, Chung F. Outcomes in day surgery. Current Opinion in Anaesthesiology 2006, 19:622-29 